\title{
PROCESOS CONCEPTUALES
}

\section{Y COGNITIVOS EN LA INTRODUCCIÓN DE LAS ECUACIONES DIFERENCIALES ORDINARIAS VÍA LA RESOLUCIÓN DE PROBLEMAS}

\section{CONCEPTUAL AND COGNITIVE PROCESSES IN THE INTRODUCTION OF ORDINARY DIFFERENTIAL EQUATIONS THROUGH PROBLEM SOLVING}

\author{
Matías Camacho Machín \\ Universidad de La Laguna \\ mcamacho@ull.es \\ Josefa Perdomo Díaz \\ Universidad de La Laguna y Centro de Modelamiento Matemático (Chile) \\ pepiperdomo@gmail.com \\ Manuel Santos Trigo \\ Cinvestav-IPN \\ msantos@cinvestav.mx
}

RESUMEN: En este artículo se identifican y analizan los procesos cognitivos que desarrollan un grupo de estudiantes de primer curso de la licenciatura en Química mientras resuelven un problema diseñado como parte de un Módulo de Enseñanza para la introducción de las Ecuaciones Diferenciales Ordinarias (EDO). La conjugación de un escenario de resolución de problemas, con el uso de tecnología (la calculadora Voyage ${ }^{\mathrm{TM}} 200$ ) y la interacción entre estudiantes se revelan como una combinación que promueve y favorece el uso de procesos que contribuyen a la construcción del concepto de Ecuación Diferencial.

PALABRAS CLAVE: Ecuaciones diferenciales ordinarias, comprensión de los conceptos, resolución de problemas, procesos cognitivos.

ABSTRACT: This study aims to analyze cognitive processes that a group of first year Chemistry students exhibit while working on a task that involves dealing with a variation phenomenon. The task is part of a teaching module to introduce the study of Ordinary Differential Equations. The implementation of the module was based on combining problem solving activities, the use of a hand-held calculator and fostering a collaboration work among students. Results indicate that the participants recognized and valued a problem solving approach that led them to use various representations and strategies to construct the concept of Differential Equation.

KEY WORDS: Ordinary differential equations, mathematical proficiency, problem solving, cognitive processes.

* Este trabajo ha sido financiado parcialmente por el Proyecto de Investigación con referencia EDU 200805254 del Ministerio de Ciencia e Innovación del Plan Nacional I+D+i y por el Proyecto con referencia 168543 del Conacyt-México.

Recibido: enero 2011 • Aceptado: setiembre 2011 


\section{INTRODUCCIÓN}

Para el aprendizaje de los conceptos matemáticos, es sabido que no es suficiente conocer una serie de definiciones y procedimientos, sino que se requiere la adquisición y el desarrollo de ciertas habilidades, capacidades y destrezas para emplear los conocimientos de manera integrada para resolver problemas, reflexionar y razonar ante determinadas situaciones matemáticas o de otros ámbitos. Además, el éxito o fracaso de los procesos de enseñanza y aprendizaje dependen en gran medida del entorno en el que se desarrollen. En este sentido, se ha constatado que, en general, los estudiantes que reciben una formación matemática centrada en el uso de definiciones y procedimientos matemáticos muestran dificultades a la hora de relacionar entre sí sus conocimientos matemáticos y utilizarlos en la resolución de problemas.

Esta problemática general se presenta en relación con distintos conceptos matemáticos, en particular, con el tratamiento de las ecuaciones diferenciales ordinarias, como muestran Camacho et al. (2007) en un estudio realizado con un grupo de alumnos que habían recibido una enseñanza de las ecuaciones diferenciales centrada en la definición formal y el uso de métodos algebraicos de resolución. En este trabajo, se analiza la forma en que los estudiantes utilizan sus conocimientos matemáticos para resolver problemas y responder a cuestiones relacionadas con las EDO. Los resultados mostraron que muchos estudiantes no establecían relaciones entre los conceptos de derivada de una función y EDO, lo que les impedía comprender, reflexionar y resolver determinadas actividades. Además, aquellos estudiantes que hacían uso del concepto de derivada de una función, en muchos casos recurrían únicamente al uso de las reglas de derivación y en muy pocas ocasiones utilizaban las representaciones gráficas para explorar significados y relaciones matemáticas (Camacho-Machín et al., 2012a y 2012b). Los estudiantes se centraron principalmente en la búsqueda de algoritmos que les permitieran resolver los problemas, mostrando serias dificultades en aquellas actividades cuyo enunciado hacía referencia a un contexto basado en una situación real (Camacho et al., 2009).

Los resultados obtenidos en esta investigación nos llevaron a plantearnos la necesidad de diseñar un Módulo de Enseñanza de las EDO que permitiera el desarrollo de habilidades y capacidades matemáticas relacionadas con la resolución de problemas de tal manera que los estudiantes adquiriesen una mayor responsabilidad en su proceso de aprendizaje. Dicho Módulo de Enseñanza está compuesto por tres problemas cuyas características generales, en relación con el contexto en el que se desarrollan, su estructura y su descripción, se presentan en la sección dedicada a la metodología de la investigación (tabla 2). Con estos problemas se introduce el concepto de EDO a partir del concepto de derivada de una función y se consideran las EDO tanto para resolverlas y obtener la expresión algebraica de las funciones solución como para analizar el comportamiento de las soluciones, cuando no se pueda resolver la EDO o la expresión de la solución no sea útil para los objetivos perseguidos. Con el diseño del Módulo de Enseñanza se pretende además promover que los estudiantes desarrollen procesos de pensamiento matemático avanzado (representación, abstracción, generalización...) y otros procesos fundamentales en la resolución de problemas matemáticos, como por ejemplo: el planteamiento de casos particulares, la verificación de respuestas y del propio proceso de resolución, etc. (Schoenfeld, 1985). Para el desarrollo y análisis de estos procesos resultó fundamental crear un ambiente de interacción entre los estudiantes, que trabajaron en parejas, y el uso de una herramienta tecnológica (la Voyage $\left.{ }^{\mathrm{TM}} 200\right)$ que permitió agilizar el proceso de resolución y plantear los problemas desde diferentes perspectivas, permitiendo así un análisis más profundo de las situaciones planteadas.

Una vez implementado el Módulo de Enseñanza en el aula, nos planteamos las siguientes preguntas que guían esta investigación: ¿qué procesos cognitivos se ven favorecidos por el uso de un modelo de enseñanza basado en la resolución de problemas y el uso de tecnología?, ¿qué aspectos conceptuales muestran los alumnos durante el desarrollo del módulo de enseñanza, en relación con el concepto de ecuación diferencial ordinaria? 
En este artículo se abordan estas cuestiones a partir del análisis del desarrollo del pensamiento matemático de los estudiantes durante el proceso de resolución del segundo de los problemas del Módulo, denominado Contaminación de mercurio. Esta actividad se considera representativa del Módulo de Enseñanza puesto que en ella se engloban los conceptos y procesos que tratan los otros dos problemas y tiene la particularidad de ser la primera en la que se explicitan diferentes etapas diseñadas para facilitar el proceso de resolución.

En resumen, los objetivos de este artículo son los siguientes: (i) describir el Módulo de Enseñanza diseñado para introducir el concepto de EDO en un ambiente de resolución de problemas y uso de tecnología, (ii) analizar los procesos cognitivos que muestran los estudiantes durante el desarrollo de dicho módulo, (iii) identificar los aspectos conceptuales que los estudiantes emplean en la resolución de los problemas, en especial aquellos relacionados con los conceptos de derivada de una función y ecuación diferencial ordinaria, y (iv) analizar el papel que jugaron el uso de tecnología y la interacción entre estudiantes en el proceso de resolución de los problemas propuestos.

\section{ANTECEDENTES}

Moreno y Azcárate (2003) señalan que, desde la perspectiva del profesor que imparte docencia en la universidad, existe la creencia de que:

Los estudiantes aprenden las ecuaciones diferenciales por imitación y memorización de situaciones y por esquemas de resolución vistos en clase; [...] son incapaces de pensar, crear y razonar por ellos mismos; [...] las definiciones son algo mecánico que tiene que aprenderse y en donde no hay nada que entender (p. 271).

Estos profesores consideran que los estudiantes, en general, «tienen un nivel de competencia y pensamiento matemático elemental, baja capacidad de razonamiento y abstracción» (p. 273). Por otra parte, reconocen que sería interesante dedicar más tiempo al estudio de modelos y menos a la resolución mecánica de ecuaciones, pero descartan hacerlo porque consideran que resulta más fácil aprender a resolver ecuaciones diferenciales que el tratamiento de modelos matemáticos.

En el campo de la Educación Matemática se han hecho distintas propuestas de modificación del modelo de enseñanza de las ecuaciones diferenciales. Una de las primeras la hace Artigue (1987), en el marco de una ingeniería didáctica en la que se potencia el uso de los sistemas de representación algebraico y gráfico. En esa investigación, los estudiantes tuvieron éxito en la resolución de determinadas actividades en las que se trabajaban simultáneamente diversos registros, sin embargo mostraron dificultades en el desarrollo de capacidades relacionadas con el tratamiento gráfico de las funciones (Artigue, 1992), lo que hace pensar que el éxito en determinadas respuestas pueda estar enmascarando dificultades de comprensión conceptual. Habre (2000) muestra cómo, alumnos que han recibido una formación del concepto de EDO con énfasis en el uso del sistema de representación gráfico, siguen considerando como primera opción, y en ocasiones única alternativa para resolver problemas, la clasificación y posterior resolución de las ecuaciones. Estos resultados avalan que los enfoques gráfico y cualitativo no generan una comprensión conceptual de manera automática sino que, por el contrario, pueden transformarse en actividades de manipulación mecánica análogas a las que se realizan al enfatizar sólo los métodos algebraicos de resolución de EDO (Rasmussen y Whitehead, 2003). Otros estudios hacen referencia a las dificultades que muestran los estudiantes en el uso de diferentes sistemas de representación y que influyen en la comprensión de conceptos básicos relacionados con las EDO (Guerrero et al., 2010; Rasmussen, 2001).

Otra propuesta de enseñanza del concepto de EDO viene formulada en el marco del proyecto Inquiry-Oriented Differential Equation (IO-DE), en el cuál se interpreta el concepto de EDO como una expresión que indica el comportamiento de una función con respecto al tiempo (Rasmussen y 
Kwon, 2007) y se promueve que los estudiantes establezcan sus propios métodos de resolución, proponiendo distintos tipos de actividades que denominan de predicción, exploración, matematización y generalización (Rasmussen y Blumenfeld, 2007). Durante las clases los estudiantes participan en discusiones, plantean conjeturas, explican y justifican sus ideas y resuelven problemas. Rasmussen et al. (2006) muestran que los estudiantes que participan en este proyecto obtienen buenos resultados en actividades relacionadas con la modelización y el análisis del comportamiento de las soluciones de una EDO y retienen los conocimientos y habilidades matemáticas (Kwon et al., 2005).

\section{MARCO CONCEPTUAL}

Para caracterizar el pensamiento matemático de los estudiantes seguiremos la propuesta del National Research Council [NRC] (2001), quienes consideran cinco elementos que los estudiantes deben desarrollar como parte del aprendizaje de las matemáticas y que la enseñanza, por tanto, debería promover ${ }^{1}$ :

- Comprensión conceptual: comprensión de los conceptos matemáticos, las operaciones y las relaciones.

- Fluidez con los procedimientos: habilidad en la ejecución de procedimientos de forma flexible, precisa, eficiente y correcta.

- Competencia estratégica: habilidad para formular, representar y resolver problemas matemáticos.

- Razonamiento adaptativo: capacidad para pensar de forma lógica, reflexionar, explicar y justificar.

- Disposición productiva: inclinación habitual para ver las matemáticas como prácticas, útiles y valiosas, acompañado de confianza en la propia eficacia y diligencia.

Estos cinco elementos deben estar interconectados, lo que refleja el hecho de que una comprensión profunda requiere que el estudiante conecte distintas piezas de conocimiento y que esa conexión es un factor importante a la hora de utilizar lo que saben, de forma productiva, en la resolución de problemas (Santos, 2007). Los conceptos de derivada de una función y de ecuación diferencial están relacionados de una forma muy estrecha, pudiendo incluso considerarse que una ecuación diferencial es uno de los usos o significados que se pueden asociar al concepto de derivada (Camacho-Machín et al., 2012a y 2012b). Esta relación es la base conceptual sobre la que se diseña el Módulo de Enseñanza y, en particular, el problema Contaminación de mercurio. La manera en que se establezcan las conexiones entre los distintos componentes del aprendizaje matemático dependerá del modelo de enseñanza planteado, como muestra el hecho de que, en una enseñanza basada en el uso de métodos algorítmicos, los estudiantes tengan dificultades para reflexionar sobre sus propios conocimientos matemáticos y utilizarlos en la resolución de problemas (Camacho-Machín et al., 2012a y 2012b). En este sentido, consideramos que, tal y como señala Santos (2007), un modelo de enseñanza basado en la resolución de problemas ofrece oportunidades para establecer conexiones razonadas entre distintos elementos matemáticos y favorece que los estudiantes desarrollen o construyan procesos asociados al Pensamiento Matemático Avanzado como abstraer, analizar, categorizar, conjeturar, generalizar o sintetizar, de la misma forma que promueve que los alumnos desarrollen habilidades como examinar, representar, transformar, resolver y aplicar, y consideren las matemáticas como una disciplina activa, a cuyos conocimientos pueden acceder e incluso construir (NCTM, 2009).

1. El NRC (2001) utiliza el término «mathematical proficiency» para referirse a estos cinco componentes del aprendizaje que engloban habilidad, competencia, conocimiento y facilidad para las matemáticas (p. 116 , correspondiente a la edición de 2009). 
En el diseño de los problemas que forman el Módulo de Enseñanza se tuvo en consideración que la resolución de un problema pasa por diferentes etapas entre las que se encuentran el análisis y la comprensión de la situación planteada y la resolución del problema particular (Polya, 1945) y la necesidad de promover en los estudiantes de los niveles universitarios el desarrollo de procesos propios del pensamiento matemático avanzado como la abstracción y la generalización y motivar que los alumnos controlen su propio proceso de solución. Todos estos elementos nos llevaron a distinguir entre las cinco etapas que Barrera-Mora y Santos-Trigo (2002) proponen: análisis y comprensión de la situación, solución del problema particular, planteamiento y solución del caso general y análisis retrospectivo del proceso de solución.

Otros elementos que se consideraron en el diseño del Módulo de Enseñanza son el uso de tecnología y la interacción entre los estudiantes. En relación con el uso de tecnología y su influencia en los procesos de enseñanza y aprendizaje, Guerrero et al. (2010) muestran que permite profundizar en el análisis de fenómenos, favoreciendo así la modelización de situaciones. Además facilita el acceso a múltiples representaciones de un mismo concepto, el desarrollo de estrategias para explorar ideas matemáticas y la comprobación de conjeturas (Santos, 2007), permitiendo que los estudiantes puedan centrarse en reflexionar acerca de los problemas planteados, tomar decisiones, razonar y resolver. En cuanto a las interacciones, Nickerson y Bowers (2008) señalan que el tipo de interacción que se produce entre el estudiante y el profesor afecta a las actitudes, los contenidos y la comprensión de los alumnos. Si bien un estudiante puede construir su propio conocimiento matemático, desarrollando un aprendizaje significativo (Santos, 2007), el trabajo en parejas puede mejorar el proceso de argumentación, las habilidades heurísticas, las formas de enfocar un problema y de generar nuevas ideas dentro del proceso de resolución (Cobo y Fortuny, 2000). De esta forma, el trabajo en parejas contribuye, en particular, al desarrollo de la comprensión conceptual y el razonamiento adaptativo puesto que el hecho de justificar y explicar sus ideas, promueve que los estudiantes clarifiquen sus conocimientos y razonamientos.

La reflexión e integración de estos elementos desembocan en el diseño de una trayectoria de aprendizaje (Simon, 1995) en la que las tareas de enseñanza propuestas son problemas en los que se consideran distintas etapas de resolución. El análisis de los conocimientos, recursos y tipos de razonamiento que los estudiantes muestran en el desarrollo de dicha trayectoria es fundamental para la selección o diseño de nuevas propuestas que desarrollen el pensamiento matemático de los estudiantes (Sacristán et al., 2010). Para analizar la complejidad del comportamiento de los estudiantes durante el proceso de resolución de problemas Schoenfeld (1985) propone cuatro categorías: los recursos matemáticos de los que dispone el estudiante (incluyendo intuiciones o conocimiento informal, procedimientos algorítmicos o rutinarios, etc), las heurísticas o técnicas de resolución de problemas que conoce y utiliza (representar gráficamente la situación, introducir notación apropiada, analizar problemas similares,...), el control o la forma en que selecciona y utiliza los recursos de que se dispone (planificación, monitoreo, evaluación, toma de decisiones,...) y el sistema de creencias acerca de las matemáticas (en relación con sí mismo, el entorno, el tópico particular tratado o la disciplina matemática).

\section{METODOLOGÍA}

En esta sección se describe, en líneas generales, el Módulo de Enseñanza diseñado para introducir el concepto de EDO y las características de los estudiantes que participaron en la investigación. A continuación se detalla el diseño del problema Contaminación de mercurio y cómo se desarrollaron las sesiones de clase dedicadas a su resolución. La sección finaliza con la descripción de la metodología utilizada para analizar los datos obtenidos. 


\section{Participantes}

Esta investigación se realizó con un grupo de 15 estudiantes de un primer curso de la licenciatura en Química que habían cursado previamente cálculo en una variable (funciones, derivación e integración), funciones de varias variables, derivación parcial y extremos de funciones de dos y tres variables. Las últimas diez sesiones del curso, cada una de ellas de una hora de duración, correspondían a la introducción del concepto de EDO y fueron las que se utilizaron para la implementación del Módulo de Enseñanza. Para el desarrollo del Módulo de Enseñanza los estudiantes se agruparon formando seis parejas y un trío (Tabla 1).

Tabla 1.

Agrupación de los participantes

\begin{tabular}{|c|c|c|c|c|c|c|}
\hline G1 & G2 & G3 & G4 & G5 & G6 & G7 \\
\hline $\begin{array}{c}\text { Sonia } \\
\begin{array}{c}\text { Alberto } \\
\text { Juan }\end{array}\end{array}$ & $\begin{array}{c}\text { Manuel } \\
\text { Ginés }\end{array}$ & $\begin{array}{c}\text { Milagros } \\
\text { Silvia }\end{array}$ & $\begin{array}{c}\text { Nicanor } \\
\text { Mar }\end{array}$ & $\begin{array}{c}\text { Virginia } \\
\text { Carmen }\end{array}$ & $\begin{array}{c}\text { Alexis } \\
\text { Zoraida }\end{array}$ & $\begin{array}{c}\text { Nieves } \\
\text { Naomi }\end{array}$ \\
\hline
\end{tabular}

\section{Módulo de Enseñanza}

El Módulo de Enseñanza está compuesto por tres problemas cuyas características generales se describen en la tabla 2. Su diseño responde a dos objetivos principales: la introducción del concepto de EDO a partir del concepto de derivada, como alternativa al uso de la definición formal, y la creación de un ambiente de resolución de problemas para favorecer el aprendizaje del concepto.

Los problemas son adaptaciones de tres situaciones utilizadas tradicionalmente en la enseñanza de las ecuaciones diferenciales: la descomposición de elementos químicos, la mezcla de sustancias y la dinámica de poblaciones. En el diseño de cada problema se incluye, además, una serie de cuestiones que guían a los estudiantes en los diferentes procesos cognitivos que se pretende activar (reflexión, representación, interpretación, resolución, generalización, verificación, etc.)

Tabla 2.

Problemas del Módulo de Enseñanza

\begin{tabular}{|c|c|c|c|}
\hline & $\begin{array}{l}\text { Desintegración del uranio } \\
\text { (1 sesión) }\end{array}$ & $\begin{array}{l}\text { Contaminación de mercurio } \\
\text { (5 sesiones })\end{array}$ & $\begin{array}{l}\text { Dinámica de poblaciones } \\
\text { (4 sesiones) }\end{array}$ \\
\hline Contexto & $\begin{array}{l}\text { Descomposición de elementos } \\
\text { químicos. }\end{array}$ & Mezcla de sustancias. & Población de peces. \\
\hline Estructura & $\begin{array}{l}\text { Planteamiento de la situa- } \\
\text { ción, seguido de una serie de } \\
\text { cuestiones. }\end{array}$ & $\begin{array}{l}\text { Planteamiento de la situa- } \\
\text { ción, seguido de seis etapas } \\
\text { de resolución en las que se } \\
\text { incluyen diferentes cuestiones } \\
\text { y actividades. }\end{array}$ & $\begin{array}{l}\text { Planteamiento de la situa- } \\
\text { ción, seguido de cinco etapas } \\
\text { de resolución en las que se } \\
\text { incluyen diferentes cuestiones } \\
\text { y actividades. }\end{array}$ \\
\hline Descripción & $\begin{array}{l}\text { Se analizan diferentes } \\
\text { situaciones de variación y sus } \\
\text { representaciones en lenguaje } \\
\text { matemático. } \\
\text { Finaliza con la introducción } \\
\text { del concepto de EDO, orden } \\
\text { y solución de la misma. }\end{array}$ & $\begin{array}{l}\text { Se obtiene la expresión de } \\
\text { una EDO partiendo de una } \\
\text { situación de variación. } \\
\text { Se analizan las representacio- } \\
\text { nes gráfica y algebraica de la } \\
\text { función solución. } \\
\text { Se generaliza la situación. }\end{array}$ & $\begin{array}{l}\text { Se obtiene la expresión de } \\
\text { una EDO partiendo de una } \\
\text { situación de variación. } \\
\text { Se analiza el comportamiento } \\
\text { de la función solución a través } \\
\text { de la EDO. } \\
\text { Se generaliza la situación. }\end{array}$ \\
\hline
\end{tabular}




\section{El problema "Contaminación de mercurio»}

Se trata de un problema de mezcla de sustancias cuya resolución pasa por el planteamiento y la resolución de una EDO de variables separables. El diseño del problema guía a los estudiantes en la construcción, análisis y generalización del modelo matemático, haciendo explícitas seis etapas de resolución.

Etapa 0: Comprensión del problema

Se pide a los estudiantes que elaboren un informe en el que tienen que explicar cuál es la situación a la que se están enfrentando, qué datos consideran relevantes y cómo creen que pueden resolver el problema. Con esta actividad se pretende que los estudiantes reflexionen acerca de la situación planteada y las posibles vías de resolución, antes de comenzar con la ruta establecida.

Etapa 1. Hacia la construcción de un modelo

El objetivo de esta etapa es que los estudiantes identifiquen la información relevante para la resolución del problema, para ello se formulan una serie de preguntas como ¿qué cantidad de mercurio entra en el estanque por cada litro de agua introducido?, el mercurio que entra en el depósito, ¿permanece siempre en él?, ¿a qué velocidad sale la disolución del estanque?, etc.

Etapa 2. Análisis de la situación

Durante esta etapa se guía a los estudiantes en el proceso de representación de la información en términos matemáticos. Siendo $p(t)$ la cantidad de mercurio que hay dentro del depósito en cualquier instante de tiempo, se pide a los estudiantes expresar en términos matemáticos la cantidad de mercurio que entra y sale del depósito por unidad de tiempo y relacionar dichas expresiones para indicar la variación de la cantidad de mercurio que hay en el depósito. Esta etapa concluye con la expresión de la EDO que modela la situación, $\frac{d p}{d t}=0^{\prime} 3-0^{\prime} 0003 p$

Etapa 3. Solución del caso particular

En esta etapa los estudiantes comienzan reflexionando acerca de los contenidos matemáticos introducidos hasta el momento, relacionándolos con la expresión obtenida al final de la etapa anterior. Para ello se plantean cuestiones como: la expresión que has obtenido al final de la etapa anterior, jes una EDO?, ¿de qué orden?, ¿qué elementos aparecen en la ecuación que hacen afirmar que se trata de una EDO y que ese es su orden?; Las EDO de primer orden están clasificadas en distintos tipos. Analiza de cuál de estos tipos es la EDO que modela nuestra situación, ¿qué caracteristicas de la misma te han hecho afirmar esto?

A continuación, el profesor de la materia explica a todo el grupo el método algebraico de resolución de las EDO de variables separables. Los alumnos resuelven la EDO obtenida en la etapa anterior empleando este método y la Voyage $\mathrm{T}^{\mathrm{TM}} 200$ y comparando los resultados obtenidos.

Esta etapa finaliza con la representación gráfica de la solución del caso particular (correspondiente al dato inicial $p(0)=0$ ) y su análisis a partir de preguntas como ¿crees que hay una cantidad máxima de mercurio que puede haber en el depósito?, ¿cuál es esa cantidad?; El cliente te ha dicho en su mensaje que, en este momento, la concentración de mercurio que hay en el depósito es de 0 '7 gramos por litro. ¿Es eso posible?

Etapa 4. Planteamiento y solución de casos generales

Esta etapa está dividida en cuatro apartados análogos, en los que se van considerando y generalizando determinados elementos que influyen en la situación planteada: la cantidad de mercurio que se introduce en el estanque $(m)$, la velocidad a la que entra y sale la disolución $(v)$, el volumen del depósito $(V)$ y la cantidad inicial de mercurio que hay en el estanque $\left(p_{0}\right)$. En cada uno de estos apartados los estudiantes deben cumplimentar una tabla en la que figuran las EDO, soluciones generales y particulares 
correspondientes a distintos valores del parámetro a generalizar (tablas 3 y 4). Las secciones finalizan con una serie de cuestiones cuyo objetivo es el análisis del modelo parcialmente generalizado que se acaba de obtener. Por ejemplo, en el primer apartado, donde se consideran diferentes valores para la cantidad de mercurio que se introduce en el depósito, se formulan preguntas como: La función que modela la situación, ¿depende de m?, ¿Y la cantidad máxima de mercurio que se puede alcanzar en el depósito? Representa gráficamente, en una misma pantalla, las funciones que modelan la situación para los casos en los que se introducen 0.1, 0.2, 0.3 y 0.4 gramos de mercurio por litro de solución. En un instante de tiempo determinado, ¿en cuál de los casos se alcanza una mayor concentración de mercurio en el depósito? ¿Qué cantidad de mercurio debemos introducir para que la concentración máxima que se alcance sea de 0'7 gramos por litro?

Tabla 3.

Primer apartado de la etapa 4

\begin{tabular}{|c|c|c|c|c|}
\hline $\begin{array}{c}\text { Cantidad } \\
\text { de } \\
\text { mercurio } \\
\text { que se } \\
\text { introduce } \\
\text { en el } \\
\text { depósito } \\
\text { (gr/) }\end{array}$ & $\begin{array}{c}\text { EDO que modela la } \\
\text { situación }\end{array}$ & Solución de la EDO & $\begin{array}{c}\text { Función que modela la situación real } \\
p(0)=0\end{array}$ & $\begin{array}{c}\text { Cantidad máxima de } \\
\text { mercurio que puede } \\
\text { haber en el depósito }\end{array}$ \\
\hline 0.1 & $\frac{d p}{d t}=0.3-0.0003 p(t)$ & $p(t)=C \cdot e^{-0.0003 t}+1000$ & $p(t)=-1000 \cdot e^{-0.0003 t}+1000$ & $1000 \mathrm{gr}$ \\
\hline 0.2 & & & & \\
\hline 0.3 & & & & \\
\hline 0.4 & & & & \\
\hline$\ldots$ & & & & \\
\hline $\mathrm{m}$ & & & & \\
\hline
\end{tabular}

Tabla 4.

Último apartado de la etapa 4

\begin{tabular}{|c|c|c|c|c|}
\hline $\begin{array}{c}\text { Cantidad } \\
\text { inicial de } \\
\text { mercurio } \\
\text { en el } \\
\text { depósito } \\
\text { (gramos) }\end{array}$ & $\begin{array}{l}\text { EDO que modela la } \\
\text { situación }\end{array}$ & Solución de la EDO & $\begin{array}{l}\text { Función que modela la situación real } \\
\qquad p(0)=p_{0}\end{array}$ & $\begin{array}{l}\text { Cantidad máxima de mercurio } \\
\text { que puede haber en el depósito }\end{array}$ \\
\hline 0 & \multirow{5}{*}{$\frac{d p}{d t}=v \cdot m-\frac{v}{V} p(t)$} & \multirow{5}{*}{$p(t)=C \cdot e^{\frac{v}{V^{t}}}+V \cdot m$} & $p(t)=-V \cdot m \cdot e^{\frac{\dot{v}}{V}}+V \cdot m$ & $V \cdot m$ \\
\hline 100 & & & & \\
\hline 500 & & & & \\
\hline$\ldots$ & & & $\ldots$ & $\ldots$ \\
\hline$P_{0}$ & & & & \\
\hline
\end{tabular}

La etapa 4 concluye proponiendo a los estudiantes una actividad cuyo objetivo es establecer si interrelacionan los dos contextos y han contestado a las cuestiones anteriores de forma razonada. Para ello se utilizan dos depósitos con características diferentes y se hacen preguntas acerca del tiempo que tardan en alcanzar una concentración determinada ${ }^{2}$.

2. El enunciado de esta actividad se presenta en la sección de análisis de los datos. 
Etapa 5. Análisis retrospectivo

El objetivo de esta última etapa del problema es que los estudiantes reflexionen sobre el proceso de resolución seguido, desde la propuesta inicial que hicieron hasta el planteamiento y la resolución de situaciones más generales. Mediante la elaboración de un informe, los alumnos mostrarán aquellos aspectos del problema y su resolución a los que han dado mayor relevancia.

Con este diseño se persigue que los estudiantes desarrollen su habilidad para formular, representar y resolver problemas matemáticos y su capacidad para reflexionar, explicar y abstraer, es decir, la competencia estratégica y el razonamiento adaptativo (NRC, 2001), a la vez que se influye en la comprensión conceptual y la disposición productiva de los alumnos. El uso de la calculadora Voyage ${ }^{\mathrm{TM}} 200$ se planteó como herramienta que facilita el tratamiento de diferentes sistemas de representación y permite que los estudiantes se centren en el análisis y la resolución del problema (Santos, 2007).

\section{Desarrollo en el aula}

Cada una de las cinco sesiones dedicadas a la resolución del problema Contaminación de mercurio comenzó con una breve puesta en común de las actividades realizadas durante la sesión anterior. A continuación se entregaba a cada grupo una calculadora y la documentación necesaria para continuar con la resolución del problema, que consistía en el conjunto de preguntas y actividades planteadas en cada una de las etapas de resolución, que eran presentadas por separado a los estudiantes.

\section{Metodología de análisis}

En cada una de las cinco sesiones dedicadas a la resolución de este problema se hizo copia de la documentación que había escrito cada uno de los siete grupos de estudiantes y del trabajo que habían realizado en la calculadora Voyage ${ }^{\mathrm{TM}} 200$. El análisis de estos materiales nos permitió identificar aquellas preguntas o actividades en las que la mayoría de los estudiantes cometían errores, así como tener una idea inicial del trabajo de cada pareja. A continuación se revisaron las grabaciones realizadas durante cada una de las sesiones a cada uno de los grupos, momento en el que pudimos analizar las reflexiones y razonamientos de los estudiantes y detectar los momentos en los que mostraban dudas.

Se distinguen seis fases para el análisis de los datos registrados, que se corresponden con las etapas consideradas en el diseño, es decir, se analizará la redacción del informe correspondiente a la actividad inicial, la expresión de la EDO que modela la situación planteada, el análisis de la representación gráfica de la función solución, el proceso de generalización al que se dedica la etapa 4 del problema y la redacción del informe final en el que los estudiantes revisan su propio proceso de resolución.

Para el análisis de los aspectos conceptuales, se considera la relación entre el concepto de derivada de una función y el de ecuación diferencial, las soluciones generales y particulares de la EDO que modela el problema, su representación gráfica y su comportamiento asintótico. Dentro de los procesos cognitivos se distingue entre procesos, procedimientos y heurísticas. Se consideran principalmente los procesos de abstracción y generalización; los procedimientos hacen referencia a métodos específicos, por ejemplo, de resolución de ecuaciones, reglas de derivación, etc. Por último, las heurísticas contemplan las diferentes maneras de buscar la solución a un determinado problema (Schoenfeld, 1985). 


\section{PRESENTACIÓN Y ANÁLISIS DE LOS RESULTADOS}

\section{Comprensión del problema}

En este primer momento del proceso de resolución se pudo observar que los estudiantes mostraron distintas interpretaciones del problema. Por ejemplo, cuatro grupos copian el enunciado sin mostrar algún análisis (G1, G5, G6 y G7), mientras que los otros dos identifican elementos importantes del enunciado y tratan de establecer relaciones. Sólo tres parejas mencionan algunas propuestas para resolver el problema (G1, G4 y G6).

En el diálogo mantenido en el grupo G4 se observa un primer intento de identificar conceptos y relaciones matemáticas en el enunciado del problema.

Mar: $\quad$... Gramos por litro... o sea, aquí lo que tenemos es la ecuación de la concentración por tiempo... la velocidad... o sea, la derivada de la concentración

Nicanor: Um... sí

Mar: $\quad$ Y si integramos tenemos la ecuación de la concentración de mercurio dependiente del tiempo.

Nicanor: ¿Cómo?

Mar: La derivada, si integras, es el camino opuesto.

Se observa que Mar utiliza el término «ecuación» para referirse a la expresión de una función que depende del tiempo, que relaciona el concepto de derivada con el de velocidad y que considera la integración como operación inversa de la derivación. Mar confunde las expresiones de la concentración de mercurio que se introduce en el estanque $\left(0^{\prime} 1 \mathrm{gr} / \mathrm{l}\right)$ y de la cantidad de dicho elemento que entra en el depósito por unidad de tiempo $\left(0^{\prime} 3 \mathrm{gr} / \mathrm{min}\right)$. Otros estudiantes también confundieron estas expresiones, lo que les llevó a responder de forma incorrecta a varias preguntas planteadas a lo largo del problema, siendo la causa principal por la que determinados estudiantes no lograron la expresión del modelo matemático.

La pareja G4 concluyó su propuesta de resolución señalando que

habría que definir una función que de cuenta de la cantidad de mercurio en el agua en cada momento [...] otra que indique la cantidad que sale [...] Hallar la variación de estas dos ecuaciones para obtener otra que nos de la concentración de mercurio por litro en cada momento.

Lo que muestra que estaban considerando un problema en el contexto matemático, al igual que el grupo G6 que propuso el uso de las «rectas de calibrado» (que no son aplicables en esta situación). El resto de los grupos asociaron el problema a resolver con un problema químico (disminuir la concentración de mercurio en el estanque) y no con un problema matemático (Imagen 5). Esta situación, en la que los estudiantes dan prioridad a argumentos basados en su experiencia relacionada con la situación real, familiar para ellos, puede influir en la forma en que los estudiantes piensan y tratan de resolver el problema (Rasmussen y Ruan, 2008). 


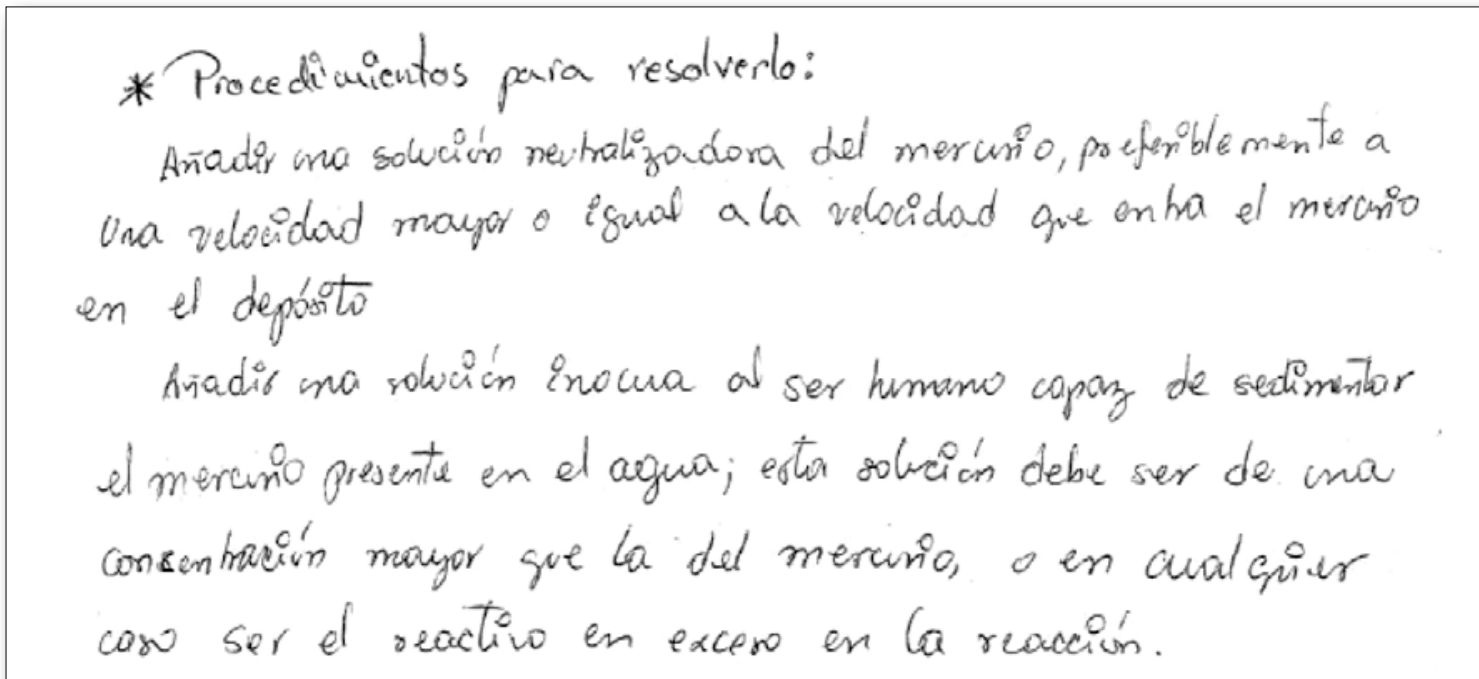

Imagen 5. G1 piensa en un problema químico

\section{Construcción del modelo matemático}

Después de la fase de comprensión, los estudiantes tenían que responder a una serie de cuestiones sobre la situación planteada y expresar cierta información en lenguaje matemático, proceso que no resulta trivial a los estudiantes (Camacho-Machín et al., 2012a y 2012b) y que es fundamental en la resolución de problemas. En el siguiente esquema (esquema 6) se esboza el proceso de representación que puede conducir a la construcción del modelo matemático, indicando con líneas discontinuas aquellos en los que los estudiantes tuvieron mayor dificultad.

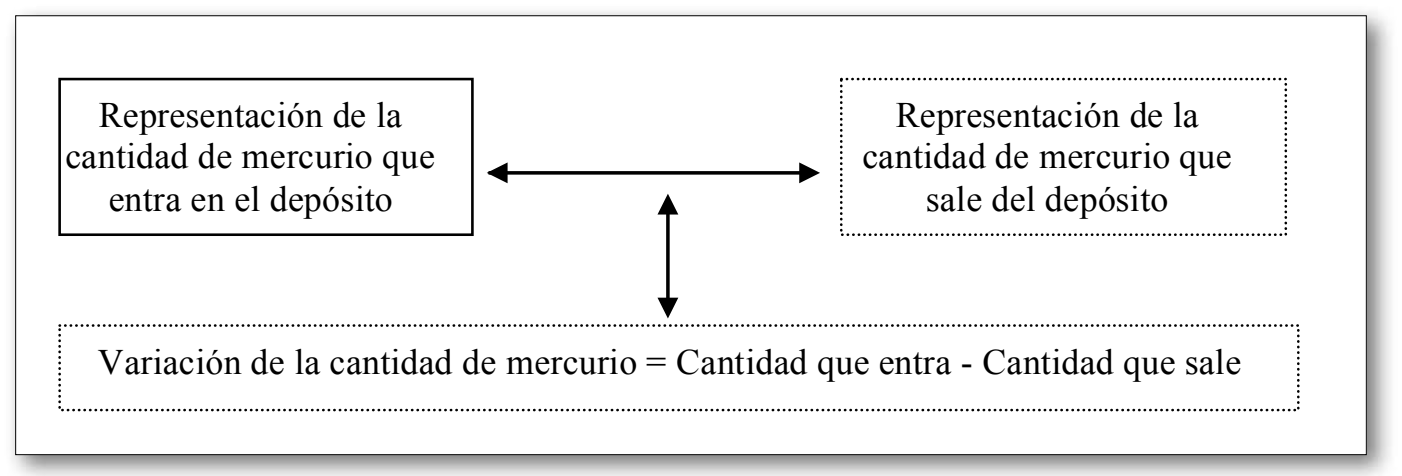

Esquema 6. Procesos de representación para obtener la EDO

Todos los grupos expresaron correctamente la cantidad de mercurio que entraba en el depósito; dos de ellos no obtuvieron la representación de la cantidad de dicha sustancia que salía del mismo y sólo tres grupos presentaron el modelo matemático utilizando una EDO (tabla 7). Los estudiantes que expresaron correctamente las cantidades de mercurio que entraban y salían del depósito por unidad de tiempo se apoyaron en las unidades de medida para realizar las operaciones necesarias. Las principales causas por las que los estudiantes dudaron en la formulación de la expresión de la cantidad de mercurio que salía del depósito fueron su dependencia de la función $p(t)$ y la confusión entre proporción y cantidad de una sustancia, tal y como le ocurrió a Mar (G4) en la fase de comprensión. 
Tabla 7.

Proceso de representación mostrado en la etapa 2

\begin{tabular}{|l|c|c|c|c|c|c|c|}
\hline & G1 & G2 & G3 & G4 & G5 & G6 & G7 \\
\hline Mercurio que entra & $\mathrm{V}$ & $\mathrm{V}$ & $\mathrm{V}$ & $\mathrm{V}$ & $\mathrm{V}$ & $\mathrm{V}$ & $\mathrm{V}$ \\
\hline Mercurio que sale & Incorrecta & $\mathrm{V}$ & $\mathrm{V}$ & $\mathrm{V}$ & $\mathrm{V}$ & Incorrecta & $\mathrm{V}$ \\
\hline EDO & & $\mathrm{V}$ & Incorrecta & & $\mathrm{V}$ & & \\
\hline
\end{tabular}

La pareja G1 consideró erróneamente que las cantidades de mercurio que entraban y salían del depósito coincidían, mientras que el grupo G6 sustituyó la función $p(t)$ en la expresión de la concentración de mercurio dentro del depósito, $\frac{p(t)}{10000}$, por el valor 0 '3, correspondiente a la cantidad de mercurio que se introduce en el estanque por minuto.

Los estudiantes, en general, mostraron dificultades para representar el fenómeno de variación asociado con el problema. Sólo dos parejas expresaron correctamente el modelo matemático, relacionando la variación de la cantidad de mercurio que se produce dentro del estanque con $p^{\prime}(t)$ y, a su vez, con la diferencia entre las cantidades de mercurio que entraban y salían del estanque, obteniendo así la EDO p’=0’3-0’0003p (tabla 7). La pareja G3 expresó la situación utilizando la ecuación $p^{\prime}=\frac{p(t)}{10000}+0^{\prime} 3-\frac{3 p(t)}{10000}$, en la que se observa que no interpretaron correctamente el significado de la variación en términos de la situación planteada. El resto de los estudiantes no llega a obtener la expresión de una EDO. Entre las causas se encuentra la falta de relación entre la variación y la función derivada (G4 y G7) o entre la variación y la diferencia entre las cantidades de mercurio que entraban y salían del estanque (G1 y G6).

El análisis de estas etapas de la resolución del problema evidencia que los elementos descritos por el NRC (2001) para caracterizar la competencia matemática deben permanecer interconectados. En este caso se ha podido observar que la capacidad de reflexionar y pensar de manera lógica (razonamiento adaptativo) ha influido en el proceso de representación mostrado por los estudiantes (competencia estratégica).

Con el fin de situar a los estudiantes en el mismo punto del proceso de modelización, se concluyó la etapa 2 (análisis de la situación) con la presentación, por parte del profesor, de la EDO que modela la situación. De esta forma, todos los estudiantes dispusieron de la expresión correcta de la ecuación para continuar con el proceso de resolución. Se constató que todos los estudiantes aceptaron la validez del modelo matemático propuesto.

Posteriormente los estudiantes clasificaron la EDO y la resolvieron utilizando el método de separación de variables y la calculadora Voyage ${ }^{\mathrm{TM}} 200$, comparando los resultados obtenidos. La construcción del modelo finaliza con la obtención de la expresión de la función solución correspondiente al dato inicial $p(0)=0$. Para ello, algunos grupos (por ejemplo, G3 y G4) hicieron uso del concepto de valor numérico de una función para calcular el valor correspondiente a la constante de integración. Esto indica que el concepto de problema de valores iniciales (o problema de Cauchy) puede ser introducido a partir del concepto de valor numérico de una función, contribuyendo así a establecer relaciones entre distintos conceptos y procesos matemáticos. Sin embargo, el hecho de que no todos los estudiantes utilizaran esta relación, hace pensar que los alumnos no establecen este nexo de manera inmediata, requiriéndose la intervención del profesor o de determinadas actividades que hagan explícitas estas relaciones. 


\section{Análisis del modelo matemático}

El uso de la calculadora Voyage ${ }^{\mathrm{TM}} 200$ facilitó la representación gráfica de la función solución particular de la EDO $\left(p(t)=-1000 \cdot e^{-0.0003 t}+1000\right)$ permitiendo que los estudiantes se centraran en el análisis de dicha función y su interpretación en el contexto de la situación hipotética planteada, así como en la exploración de ideas matemáticas y la formulación de conjeturas y no en la construcción de la gráfica (Santos, 2007).

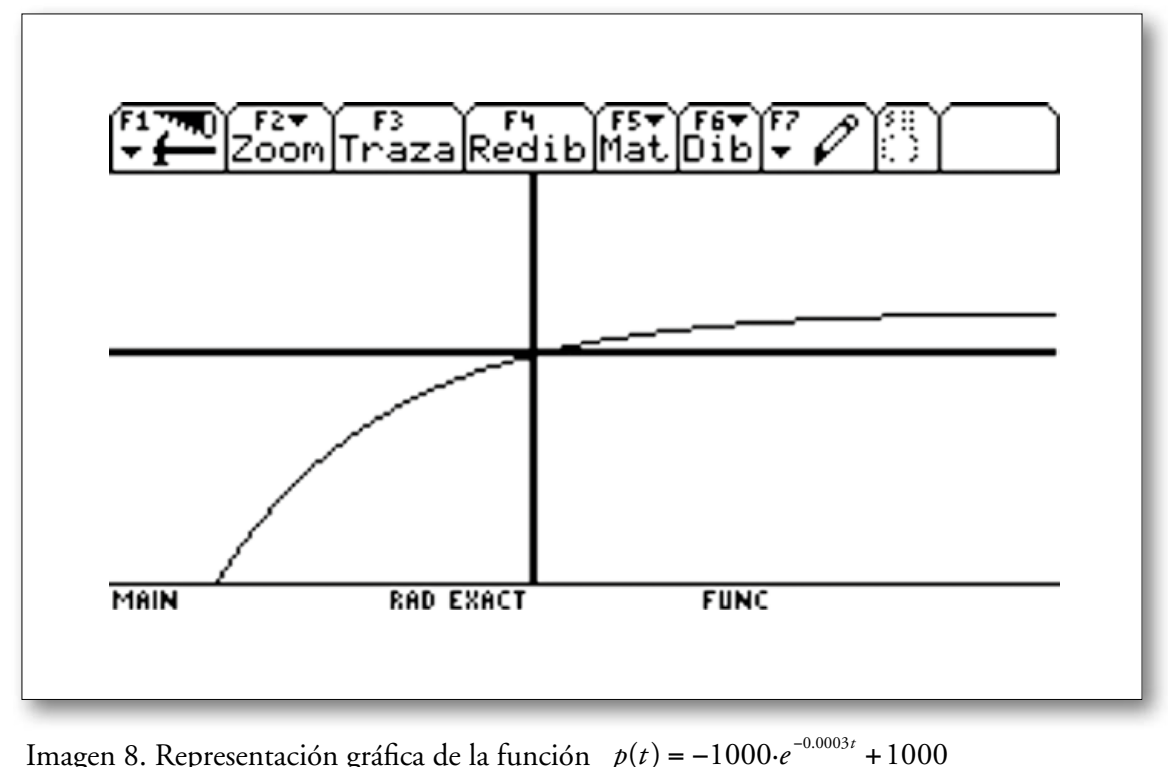

Tres de los grupos (G1, G2 y G6) interpretaron erróneamente la gráfica de la función y afirmaron que la cantidad de mercurio seguía aumentando de forma indefinida. Tampoco relacionaron el comportamiento asintótico con el cálculo del límite de la función. La pareja G3 aunque inicialmente no identificó la asíntota, lo hacen después de interactuar con el grupo G4 y calcular el valor del límite de la función en el infinito. Esta es la única pareja que realizó este cálculo sin ayuda de la Voyage ${ }^{\mathrm{TM}} 200$, lo que evidencia su fluidez en el uso de los procedimientos de cálculo (Imagen 9). Otros dos grupos (G4 y G5) utilizaron este proceso para comprobar el resultado obtenido con la calculadora.

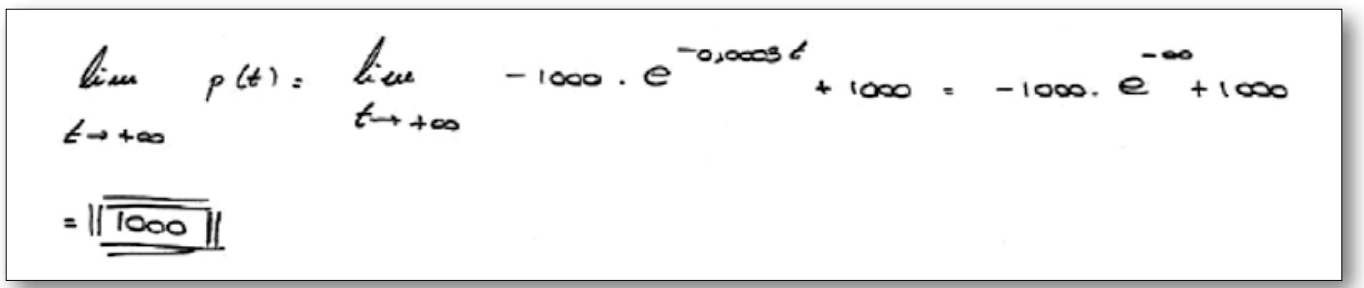

Imagen 9. Procedimiento de cálculo del límite de G3

\section{Interpretación del modelo}

Durante el desarrollo del problema se planteó a los estudiantes una cuestión para analizar la manera en que interpretan el modelo matemático obtenido durante la resolución del problema: 
Si la concentración máxima de mercurio que permite el Ministerio de Sanidad y Consumo es de 0'04 gramos por litro, ¿en qué instante de tiempo se deberá cerrar la entrada y la salida de solución al estanque?

La mayoría de los grupos respondieron correctamente. El proceso seguido fue: obtener en primer lugar la cantidad de mercurio correspondiente a una concentración de 0’04gr/l, sustituir dicho valor en la expresión de la función solución y obtener el valor del tiempo. Todos los estudiantes optaron por utilizar la Voyage ${ }^{\mathrm{TM}} 200$ para realizar estas operaciones. En esta tarea se refleja la importancia que tiene el control del propio proceso de resolución de problemas (Schoenfeld, 1985), como muestra el hecho de que los grupos G1 y G2 comenzaran planteando una regla de tres, que descartaron posteriormente. La utilidad de este proceso metacognitivo depende fuertemente de aspectos cognitivos, como se desprende del hecho de que el grupo G2, aún habiendo reflexionado sobre su proceso de resolución, respondiera de manera incorrecta a la cuestión planteada, confundiendo la concentración de mercurio con la cantidad de dicho elemento que hay dentro del estanque (Imagen 10). Vemos con esto la necesidad de que los distintos elementos que componen el aprendizaje de las matemáticas permanezcan interrelacionados (NRC, 2001).

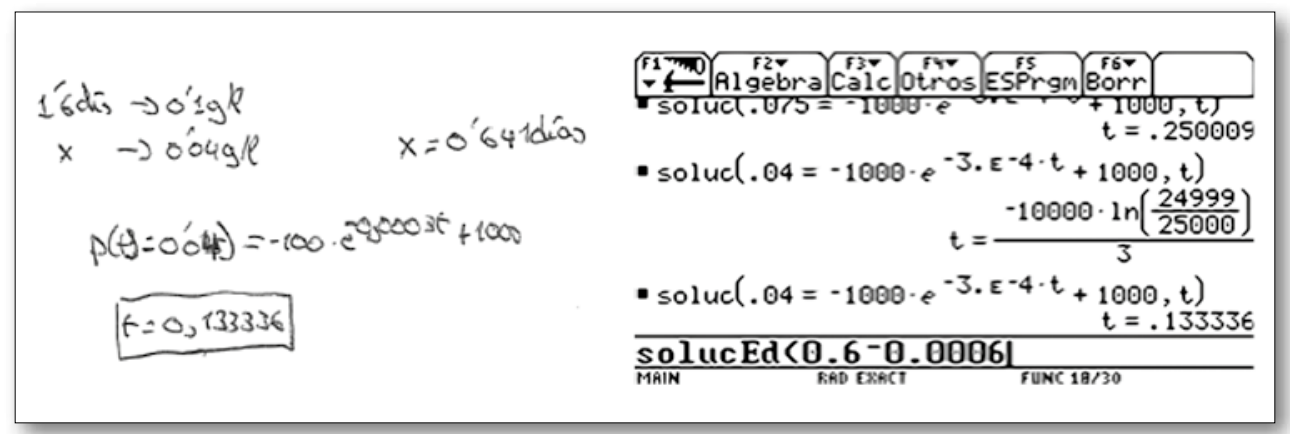

Imagen 10. Proceso de resolución de Manuel y Ginés en la interpretación del modelo

\section{Generalización del modelo}

El proceso de generalización es característico del Pensamiento Matemático Avanzado (Azcárate y Camacho, 2003). La forma en que los estudiantes se aproximen a la generalización de la situación da muestras de su habilidad en la resolución de problemas, su capacidad para abstraer y su fluidez en el uso de procedimientos matemáticos.

En la etapa de generalización del problema Contaminación de mercurio se consideraron cuatro parámetros (cantidad de mercurio que se introduce en el estanque, velocidad de entrada y salida de la disolución, volumen del depósito y cantidad inicial de mercurio que hay dentro del depósito). Los tres primeros influyen en la expresión de la EDO que modeliza la situación; el último sólo interviene en la expresión de la función solución para cada caso particular. Para el desarrollo de este proceso es fundamental reflexionar acerca del significado de las diferentes expresiones del modelo matemático (la EDO, cada uno de sus términos y las soluciones general y particular de la ecuación) en el contexto de la situación planteada.

Todos los estudiantes (excepto los del grupo G6) identificaron cada uno de los términos de la EDO con el significado asociado en la situación planteada. En el caso del parámetro correspondiente a la cantidad inicial de mercurio que hay dentro del depósito, sólo dos parejas lo relacionaron con la constante de integración que aparece en la expresión de la solución general de la EDO (tabla 13). La siguiente imagen presenta, a modo de ejemplo, la reflexión hecha por la pareja G3 para obtener la EDO correspondiente al caso en que se introducen 0’2gr/l de mercurio en el estanque (Imagen 11). 


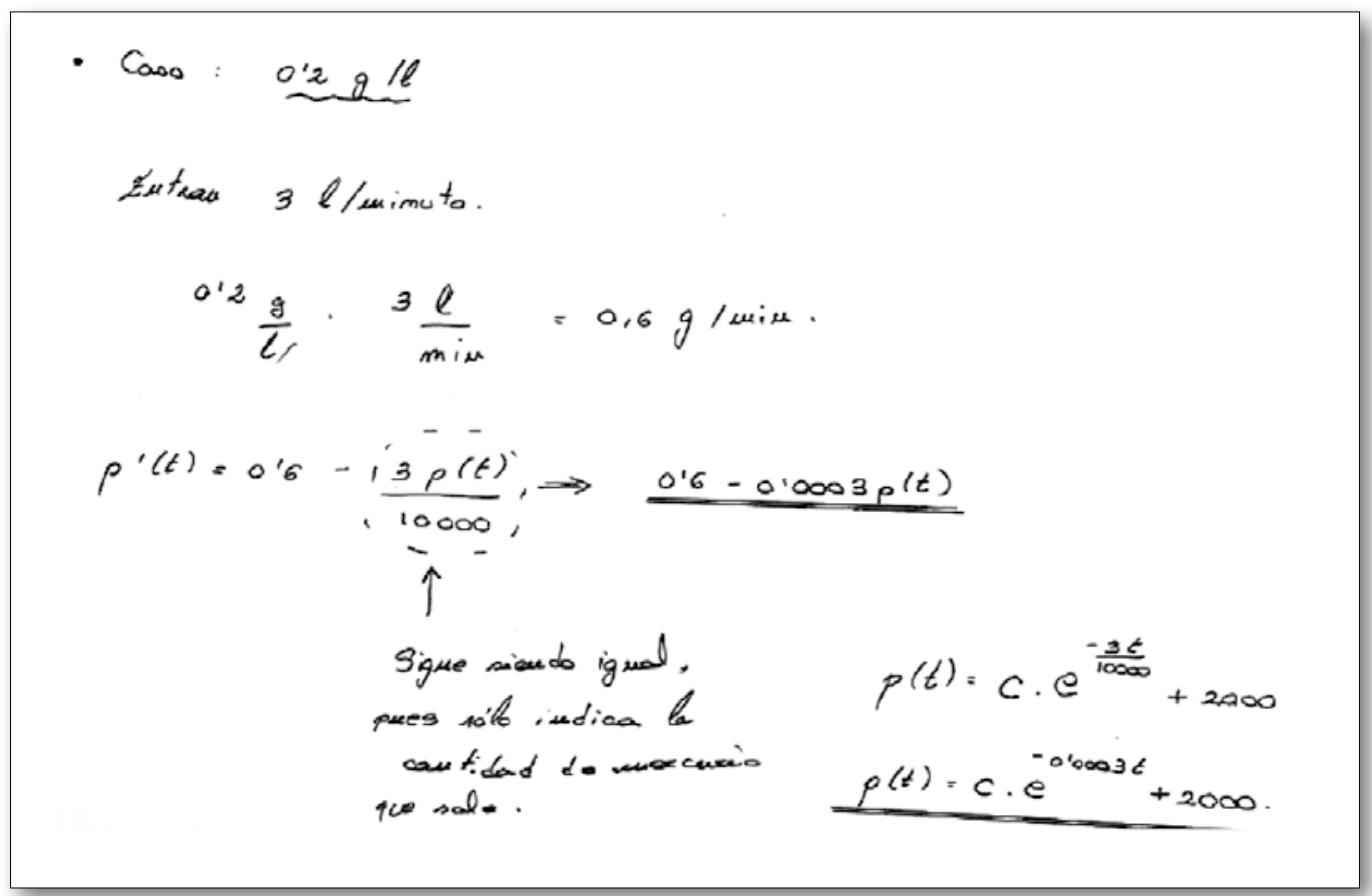

Imagen 11. Procesos de reflexión y explicación de G3

La pareja G6 generalizó las ecuaciones de manera lineal con respecto a la variación en el dato modificado, sin tener en cuenta el significado de cada uno de los términos de la EDO en la situación planteada (Imagen 12).

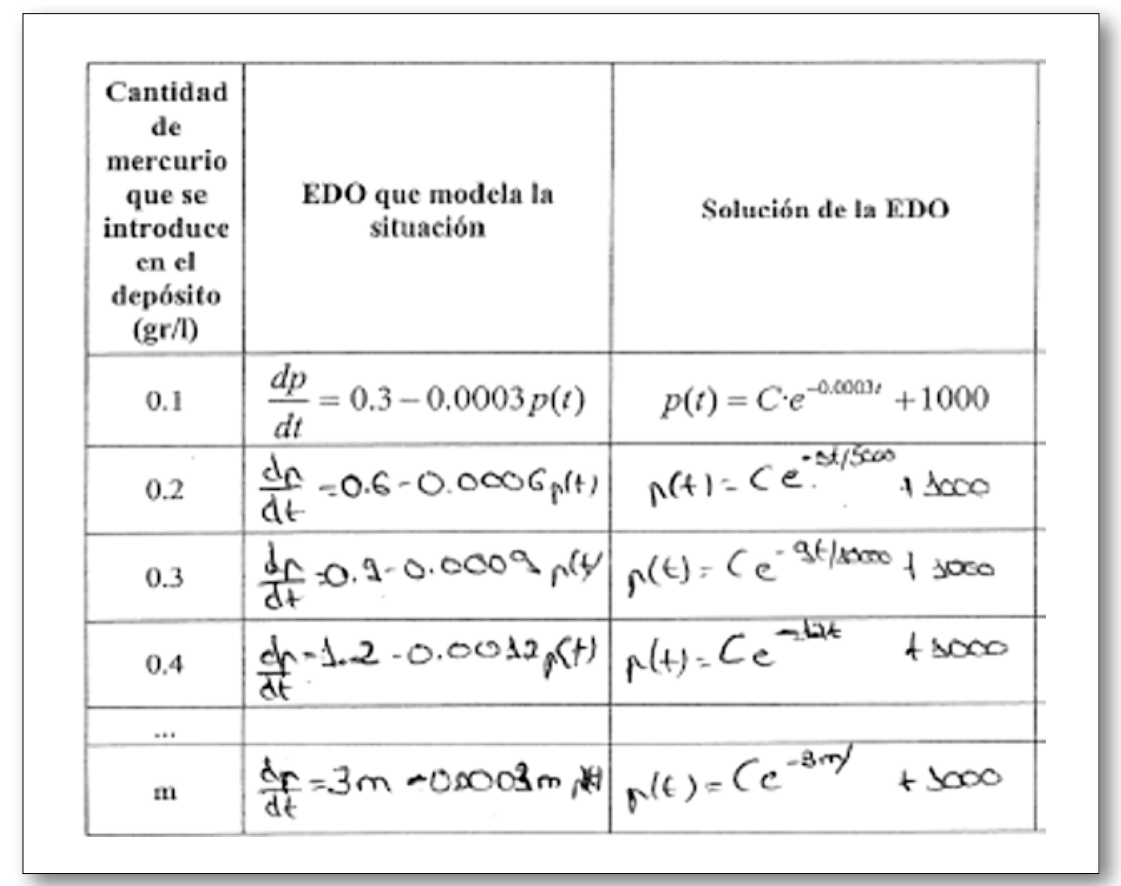

Imagen 12. Proceso de generalización de Alexis y Zoraida (G6) 
La pareja G6 posee escasos recursos para la resolución de problemas, en lo que a heurísticas se refiere, dado que la estrategia que utilizaron para completar las tablas propuestas en la etapa 4 del problema (por ejemplo la tabla 3) fue resolver cada caso por separado (al igual que G7). El resto de los estudiantes optó por resolver una o dos filas y buscar el patrón que les permitía expresar las ecuaciones y funciones que necesitaban (tabla 13). Por otra parte, sólo dos de estas parejas comprobaron cada una de las expresiones que proponían como solución, resolviendo cada EDO con la Voyage ${ }^{\mathrm{TM}} 200$ y verificando que obtenían la misma expresión que habían propuesto.

Tabla 13.

Heurísticas y control mostrado durante el proceso de generalización

\begin{tabular}{|l|c|c|c|c|c|c|c|}
\hline & G1 & G2 & G3 & G4 & G5 & G6 & G7 \\
\hline $\begin{array}{l}\text { Relación entre la EDO } \\
\text { y la situación }\end{array}$ & $\mathrm{V}$ & $\mathrm{V}$ & $\mathrm{V}$ & $\mathrm{V}$ & $\mathrm{V}$ & & $\mathrm{V}$ \\
\hline $\begin{array}{l}\text { Relación entre la cons- } \\
\text { tante de integración y la } \\
\text { condición inicial }\end{array}$ & & $\mathrm{V}$ & $\mathrm{V}$ & $\mathrm{V}$ & & & \\
\hline Búsqueda de patrones & $\mathrm{V}$ & $\mathrm{V}$ & $\mathrm{V}$ & $\mathrm{V}$ & & \\
\hline Comprobación & & & $\mathrm{V}$ & $\mathrm{V}$ & & \\
\hline
\end{tabular}

La etapa de generalización de este problema (etapa 4) finalizaba con una actividad en la que los estudiantes debían comparar dos depósitos de mercurio, con el objetivo de que interpretasen el modelo general obtenido. El enunciado de la actividad es el siguiente:

Supongamos que tenemos dos depósitos, el primero con un volumen de 60.000 litros en el que introduce una solución con 0 '2 gramos de mercurio por litro, y el segundo con un volumen de 45.000 litros en el que se introduce una solución con O'6 gramos de mercurio por litro.

Si en cada depósito tenemos una cantidad inicial de 250 gramos de mercurio y las soluciones entran y salen de ambos depósitos a una velocidad de 3 litros por minuto, ¿En cuál de los depósitos se alcanza antes una concentración de mercurio de O'1grll? ¿En qué instante de tiempo se alcanza?

En esta etapa, los grupos G1, G6 y G7 muestran una disposición poco productiva hacia las matemáticas, factor que influye en el desarrollo del aprendizaje de la disciplina (NRC, 2001), puesto que no resolvieron esta actividad durante la sesión de clases y tampoco en casa, habiéndoles dado la oportunidad de hacerlo. El grupo G2 no resolvió correctamente esta actividad por dos razones: durante todo el proceso de resolución confundían la concentración de mercurio con la cantidad de dicho elemento que había en el estanque en cualquier instante de tiempo y habían obtenido una expresión errónea para la EDO y la función que modelizan la situación general, al no relacionar la constante de integración con la condición inicial del problema (Imagen 14). 
- Si en el momento de introducir la solución con mercurio en el depósito, éste tuviera dentro 100 gramos de mercurio, ¿cuáles serían la EDO y la función que modelan la situación?

$$
\begin{aligned}
& \frac{d P}{d t}=v(100+m)-\frac{v}{V} p(t) \\
& p(t)=C e^{-(v / v) t}+V \cdot(100+m)
\end{aligned}
$$

- ¿Y si el depósito tuviera 500 gramos de mercurio?

$$
\frac{d p}{d t}=v(s 00+m)-\frac{v}{V} p(t)
$$

Imagen 14. Proceso de generalización de la condición inicial por parte de G2

El resto de las parejas interpretó correctamente el modelo matemático general, aunque la pareja G3 cometió un error al reescribir la expresión de la función que modeliza la situación, que les llevó a responder erróneamente a la actividad (Imagen 15).

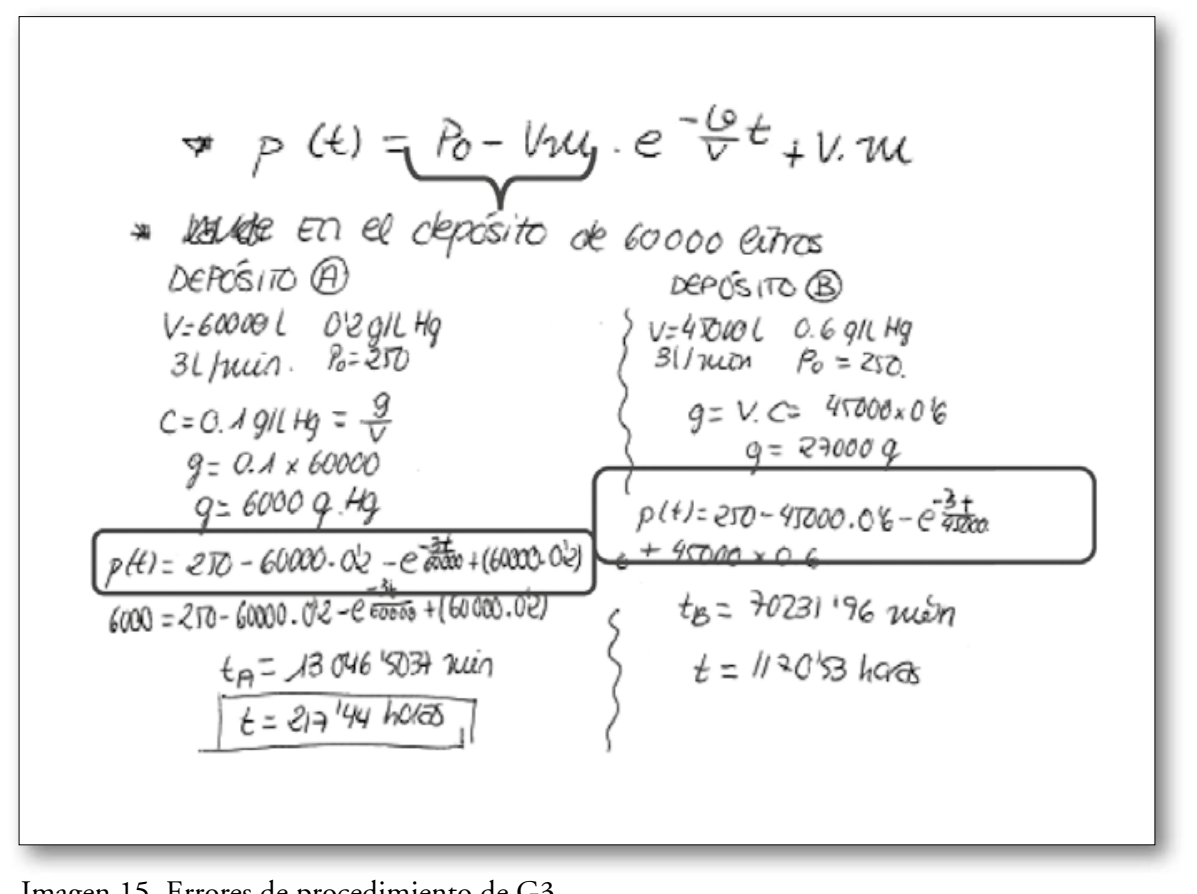

Imagen 15. Errores de procedimiento de G3

\section{Análisis retrospectivo del proceso de solución}

El informe redactado por la pareja G1 presenta los aspectos de su proceso de resolución que se han ido observando. La ausencia de reflexión y análisis de la situación les lleva a confundir la concentración y la cantidad de mercurio que hay dentro del estanque y a obviar el dato correspondiente a la velocidad de salida de la disolución del estanque (Imagen 16). 
- Dates relevantes:

Los datos más importantes que debonos tener en cuenta son:

los 1000 litros de agua, los ot gramos de mercurio que introducimos por

litro, a razón de tres litiros par auincito.

- ¿Cuál es la jucición que le permite analizar diferentes situaciones ancilogas a la que te presenta, explicándole el signaficado de los distintos elecuentos que aparecen en la expresión de ka junción?

$$
\begin{aligned}
& \frac{d p}{d t}=\theta_{m}-\frac{\theta}{v} p(t) \\
& p(t)=C e^{-\frac{v}{v} t}+v_{m}
\end{aligned}
$$

$v=$ velocidad de entrada y saliala.

$v=$ rolumen del depósito o estanque.

$m=$ cantidad de wercurio gee se intoduce.

$C=$ constanite.

$P(t)=$ concentración en cualquier instante de tiempo.

Imagen 16. Parte del análisis retrospectivo de G1

Naomi y Nieves (G7) y Manuel ${ }^{3}(\mathrm{G} 2)$ entregaron un informe individual en el que se puede observar que no reflexionan sobre todos los aspectos considerados en el proceso de resolución, en particular, en el proceso de generalización. Los informes de las parejas G3 y G4 contemplan tanto el caso particular como el general, indicando los factores que intervienen en la situación hipotética. Existe una diferencia en cuanto a la redacción de informes, relativa al papel de la EDO en el proceso de resolución. La pareja G3 indica que para resolver la situación, «analizan la variación de la cantidad de mercurio con el tiempo», obteniendo una EDO. El grupo G4 manifiesta que "la cantidad de mercurio presente en el estanque era la variación entre la cantidad de mercurio que se introducía en el estanque y la que salía de él» y que "para hallar la concentración de mercurio en el depósito hay que determinar qué cantidad de mercurio sale y entra del depósito en cada tiempo, y hallar su variación». Desde el punto de vista del significado que cada una de estas parejas ha dado a la EDO, la pareja G3 considera la ecuación diferencial como una forma de expresar información de la que disponen, mientras que el grupo G4 parece verlo como algo que hay que calcular.

\section{DISCUSIÓN Y CONSIDERACIONES FINALES}

En este artículo hemos presentado un Módulo de Enseñanza propuesto para introducir el concepto de EDO a partir de su relación con el concepto de derivada y en un ambiente que promueve el desarrollo de habilidades y capacidades útiles para la resolución de problemas matemáticos, que incluye el uso de tecnología y la interacción entre los estudiantes, que pasan a ser los protagonistas y principales responsables de su proceso de aprendizaje.

El Módulo de Enseñanza se desarrolla en un ambiente de resolución de problemas, en el que los estudiantes pasan por diferentes etapas: comprensión del problema, construcción, análisis, interpre-

3. Ginés no realiza esta actividad. 
tación y generalización del modelo matemático y análisis retrospectivo del proceso de resolución. La evolución del proceso de resolución mostrado por los estudiantes en el transcurso de estas etapas refleja algunos aspectos importantes relacionados con el desarrollo del pensamiento matemático:

1. Los estudiantes partieron considerando que el problema que debían resolver era un problema químico en lugar de matemático, y propusieron soluciones basadas en argumentos de tipo empírico. Este tipo de situaciones muestra que, el uso de problemas planteados en un contexto real, hace que determinados alumnos basen sus argumentos en lo que esperan que ocurra en la situación real y no en la información que les aporta el modelo matemático (Rasmussen y Ruan, 2008). El diseño del problema Contaminación de mercurio facilitó la transición hacia el modelo matemático puesto que los estudiantes, en ningún otro momento del proceso de resolución, muestran el uso de razonamientos empíricos que no concuerden con el modelo matemático tratado. La relación entre el contexto en que se plantea la situación y el modelo matemático se fortalece a partir de las diferentes actividades propuestas para interpretar el modelo matemático en el contexto del problema.

2. En esa transición del contexto hipotético en el que se plantea el problema hacia el lenguaje matemático, juega un papel central el proceso de representación. En Camacho-Machín et al. (2012a, 2012b) se muestran evidencias de que este proceso no resulta trivial a los estudiantes. La estructura del problema contribuyó a que algunas de las parejas participantes en esta investigación (G2 y G5) consiguieran expresar el modelo matemático, utilizando el concepto de derivada. El resto de los estudiantes no obtuvo la expresión de la EDO debido, fundamentalmente, a dificultades en la interpretación del concepto de variación. Algunos, como la pareja G7, no asociaron este significado con el concepto de derivada de una función; otros, como el grupo G6, lo asociaron con la derivada de una función pero no con la diferencia entre las cantidades de mercurio que entran y salen del depósito y otros, como la pareja G3 consideraron que la variación se correspondía con la cantidad de mercurio que había dentro del estanque.

3. En relación con el análisis del modelo matemático construido, se ha constatado que algunos estudiantes no reconocieron la asíntota horizontal en la representación gráfica de la función solución, mostrando deficiencias en el tratamiento del sistema de representación gráfico, resultados que concuerdan con los de otros autores (p.e. Artigue, 1992; Guerrero et al., 2010; Rasmussen, 2001).

4. En el proceso de generalización, la mayor dificultad estuvo en la modificación de las condiciones iniciales; si bien algunos estudiantes asociaron directamente esta información con el concepto de valor numérico de una función y el cálculo de la constante de integración (por ejemplo el grupo G4), otros consideraron que este aspecto modificaba la expresión de la EDO, obteniendo así un modelo general erróneo.

En relación con los objetivos de esta investigación, el análisis de los datos recabados ha mostrado que tanto el diseño de la actividad como el ambiente en el que se desarrolló el Módulo de Enseñanza favorecieron el uso de procesos matemáticos. Así, el diseño de la actividad permitió hacer explícitos procesos como los de análisis, representación, resolución, interpretación, generalización o abstracción. Las interacciones entre los estudiantes favorecieron los procesos de reflexión y análisis, además de los procesos de argumentación y la generación de ideas dentro de la resolución de un problema, tal y como señalan Cobo y Fortuny (2000). Por último, de acuerdo a lo señalado por Santos (2007), el uso de la herramienta tecnológica en el proceso de resolución facilitó que los estudiantes pudieran explorar la situación a través de diferentes representaciones y que se centraran en los procesos de reflexión y análisis de los modelos matemáticos, lo que resultó especialmente útil en el proceso de generalización del modelo. 
En el terreno conceptual (objetivo (iii) de la investigación), se ha podido comprobar que el diseño del problema promovió que los estudiantes obtuvieran la expresión de una EDO, partiendo del significado de la derivada como elemento que describe la variación de una función con respecto a la variable independiente. Además, les hizo reflexionar sobre la expresión matemática obtenida y los elementos que la caracterizan, previamente a considerar el proceso de resolución. La situación hipotética planteada y las actividades que se incluyeron como parte del diseño del problema permitieron presentar otros conceptos matemáticos y utilizarlos en la resolución del problema. Entre dichos conceptos se encuentran el de función, asíntota o límite, analizados a partir de representaciones gráficas y algebraicas.

En cuanto al último de los objetivos, podemos concluir que las interacciones entre los estudiantes se revelaron como una herramienta de control del conocimiento, además de promover procesos como la reflexión y la argumentación. El análisis de los datos facilitó la detección tanto de los procesos matemáticos utilizados por los estudiantes como de algunos errores de concepción, la mayoría de los cuáles fueron resueltos entre los mismos alumnos.

Finalmente, el uso de la tecnología resultó fundamental para el desarrollo de procesos del pensamiento matemático avanzado como, por ejemplo, el proceso de generalización, al permitir que los estudiantes indagaran acerca de posibles modelos matemáticos más generales, formularan conjeturas y verificaran las hipótesis formuladas durante el proceso de resolución del problema. El uso de la calculadora Voyage ${ }^{\mathrm{TM}} 200$ facilitó, además, que los alumnos se centraran en el análisis del modelo matemático a partir de la representación gráfica de la función solución de un problema de valores iniciales y que establecieran relaciones entre diferentes representaciones de un mismo concepto matemático. De esta forma, la tecnología se convirtió en un elemento natural dentro del proceso de resolución de problemas.

\section{REFERENCIAS BIBLIOGRÁFICAS}

ARTIGUE, M. (1987). Ingenierie didactique a propos d'equations differentielles. En J. Bergeron, N. Herscovics y C. Kieran (Eds.), Proceedings of the eleventh international conference of Psychology of Mathematics Education, pp. 236-242. Montreal: Université de Montréal.

ARTIGUE, M. (1992). Functions from an algebraic and graphic point of view: cognitive difficulties and teaching practices. En G. Harel y E. Dubinsky (Eds.), The concept of function. Cognitive dificulties and teaching practices. Aspects of epistemology and pedagogy, MAA Notes 25 pp. 109-132. USA: MAA.

AZCÁRATE, C. y CAMACHO, M. (2003). Sobre la Investigación en Didáctica del Análisis Matemático. Boletín de la Asociación Matemática Venezolana, 10 (2), pp. 135-149.

BARRERA-MORA, F. y SANTOS-TRIGO, M. (2002). Cualidades y procesos matemáticos importantes en la resolución de problemas: un caso hipotético de suministro de medicamento. Matemáticas Aplicadas y su Enseñanza. Serie Bachillerato, 2, pp. 8-37. México: Grupo Editorial Iberoamericana.

CAMACHO, M., PERDOMO, J. y SANTOS-TRIGO, M. (2007). La resolución de problemas en los que interviene el concepto de Ecuación Diferencial Ordinaria: Un estudio exploratorio. En M. Camacho, P. Flores y P. Bolea (Eds.), Investigación en Educación Matemática. Comunicaciones de los grupos de investigación. XI Simposio de la SEIEM pp. 87-106. Tenerife.

CAMACHO, M., PERDOMO, J. y SANTOS-TRIGO, M. (2009). Revisiting university students' knowledge that involves basic differential equation questions, PNA 3(3), pp. 23-133.

COBO, P. y FORTUNY, J. (2000). Social Interactions and Cognitive Effects in Contexts of Area-Comparison Problem Solving. Educational Studies in Mathematics, 42, pp. 115-140. 
CAMACHO-MACHÍN, M.; PERDOMO-DÍAZ, J. y SANTOS-TRIGO, M. (2012a) An Exploration of Students' Conceptual Knowledge Built in a First Ordinary Differential Equations Course (Part I), The Teaching of Mathematics, 15 (1). 1-20. http://elib.mi.sanu.ac.rs/pages/browse_issue.php?db=tm\&rbr=28

CAMACHO-MACHÍN, M.; PERDOMO-DÍAZ, J. y SANTOS-TRIGO, M. (2012b) An Exploration of Students' Conceptual Knowledge Built in a First Ordinary Differential Equations Course (Part II), The Teaching of Mathematics, 15 (2). http://elib.mi.sanu.ac.rs/pages/browse_issue.php? $\mathrm{db}=\mathrm{tm} \& \mathrm{rbr}=28$ GUERrERO, C., CAMACHO, M. y MEjÍA, H. (2010). Dificultades de los estudiantes en la interpretación de las soluciones de Ecuaciones Diferenciales Ordinarias que modelan un problema. Enseñanza de las Ciencias, 28(3), pp. 341-352.

HABRE, S. (2000). Exploring Students' Strategies to Solve Ordinary Differential Equations in a Reformed Setting. Journal of Mathematical Behavior, 18 (4), pp. 455-472.

KWON, O.N., RASMUSSEN, C. y ALLEN, K. (2005). Students' Retention of Mathematical Knowledge and Skills in Differential Equations. School Science and Mathematics, 105 (5), pp. 1-13.

MORENO MORENO, M. y AZCÁRATE GIMÉNEZ, C. (2003). Concepciones y creencias de los profesores universitarios de matemáticas acerca de la enseñanza de las ecuaciones diferenciales. Enseñanza de las Ciencias, 21(2), pp. 265-280.

NATIONAL COUNCIL OF TEACHERS OF MATHEMATICS. (2009). Focus in High School Mathematics. Reasoning and Sense Making. USA: NCTM.

NATIONAL RESEARCH COUNCIL. (2001). Adding It Up: Helping Children Learn Mathematics. J. Kilpatrick, J. Swafford, y B. Findell (Eds.). Mathematics Learning Study Committee, Center for Education, Division of Behavioral and Social Sciences and Education. Washington, DC: National Academy Press.

NICKERSON, S. y BOWERS, J. (2008). Examining Interaction Patterns in College-Level Mathematics Classes: A Case Study. En M. Carlson y C. Rasmussen (Eds.), Making the Connection. Research and Teaching in Undergraduate Mathematics Education (179-190), MAA Notes \#73. Washington, DC: MAA.

POlYA, G. (1945). How to Solve It. Princeton: Princeton University Press.

RASMUSSEN, C. (2001). New directions in differential equations. A framework for interpreting students' understandings and difficulties. Journal of Mathematical Behavior, 20, pp. 55-87.

RASMUSSEN, C. y BLUMENFELD, H. (2007). Reinventing solutions to systems of linear differential equations: A case of emergent models involving analytic expressions. Journal of Mathematical Behavior, 26, pp. 95-210.

RASMUSSEN, C. y KWON, O. (2007). An inquiry-oriented approach to undergraduate mathematics. Journal of Mathematical Behavior, 26, pp. 89-194.

RASMUSSEN, C., KWON, O., ALLEN, K., MARRONGELle, K. y BURTCH, M. (2006). Capitalizing on Advances in Mathematics and K-12 Mathematics Education in Undergraduate Mathematics: An Inquiry-Oriented Approach to Differential Equations. Asia Pacific Education Review, 7(1), pp. 85-93. RASMUSSEN, C. y RUAN, W. (2008). Teaching for Understanding: A Case of Students Learning to Use the Uniqueness Theorem as a Tool in Differential Equations. En M. Carlson y C. Rasmussen (Eds.), Making the Connection. Research and Teaching in Undergraduate Mathematics Education. MAA Notes 73, pp. 153-163. Washington, DC: MAA.

RASMUSSEN, C. y WHITEHEAD, K. (2003). Learning and Teaching Ordinary Differential Equations. In A. Selden y J. Selden (Eds.), MAA Online Research Sampler. (http://www.maa.org/t_and_1/sampler/rs_7.html) 
SACristán, A., CAlder, N., rojano, T., SAntos-Trigo, M., Friedlander, A. y meisSner, H. (2010). The Influence and Shaping of Digital Technologies on the Learning-and Learning Trajectories-of Mathematical Concepts. En C. Hoyles and J. B. Lagrange (Eds.), Mathematics Education and Technology-Rethinking the Terrain, pp. 179-226. Berlin:Springer

SANTOS, L. M. (2007). La resolución de problemas matemáticos: fundamentos cognitivos. México D.F.: Trillas.

SCHOenfeld, A. (1985). Mathematical Problem Solving. Orlando: Academic Press.

SIMON, M. (1995). Reconstructing mathematics pedagogy from a constructivist perspective. Journal for Research in Mathematics Education, 26 (2), pp.114-145.

\section{ANEXO 1}

\section{Contaminación de mercurio}

\section{A la atención del Colegio Oficial de Químicos de Canarias}

Acabamos de recibir el último informe del Ministerio de Sanidad y Consumo sobre la calidad del agua que proviene de nuestros estanques de tratamiento. Uno de ellos, el más antiguo, no cumple con los estándares recomendados.

En el estanque hay 10.000 litros de agua y estamos introduciendo en él una solución que contiene 0'1 gramos de mercurio por litro, a razón de tres litros por minuto. Con esta operación, hemos contaminado el agua del depósito, la cuál sale del estanque también a una velocidad de tres litros por minuto.

A partir del informe del Ministerio, hemos reducido la cantidad de mercurio que bombeamos en el estanque, pero me temo que no la hemos reducido lo suficiente puesto que la concentración actual es de 0'7 gramos por litro. Necesito un modelo que me permita calcular cuánto mercurio hay en el estanque en cada momento, para poder así controlar la contaminación. 


\title{
CONCEPTUAL AND COGNITIVE PROCESSES IN THE INTRODUCTION OF ORDINARY DIFFERENTIAL EQUATIONS THROUGH PROBLEM SOLVING
}

\author{
Matías Camacho Machín, Josefa Perdomo Díaz \\ Universidad de La Laguna \\ mcamacho@ull.es•pepiperdomo@gmail.com \\ Manuel Santos Trigo \\ Cinvestav-IPN \\ msantos@cinvestav.mx
}

The aim of this study is to identify and analyse cognitive processes developed by a group of students in their first year of a chemistry degree course while solving a task designed, as part of a Teaching Module (TM), to introduce Ordinary Differential Equations (ODE).The Teaching Module combines problem solving with the use of technology in an environment of interaction among students.

To structure the study, a conceptual framework was set up in which the students' mathematical thinking is characterized in terms of Mathematical proficiency ([NRC], 2001). From this point of view, the following five intertwined strands are used, which students have to develop as part of learning mathematics and should be promoted when teaching: conceptual understanding, procedural fluency, strategic competence, adaptive reasoning and productive disposition. These elements are the conceptual basis on which the Teaching Module was designed. Another important element in the conceptual framework is the consideration of interaction as an element affecting the attitudes, the content and the students' understanding (Nickerson and Bowers, 2008).

Based on the conceptual framework, we designed a possible learning route by considering a task (Mercury Pollution) that was structured following the five steps proposed by Barrera-Mora and Santos-Trigo (2002): analysis and understanding of the situation, solution of the particular problem, approach and solution of the general case and retrospective analysis of the solution process. This learning route was implemented in a group of 15 first year students in the chemistry degree course and took place in the last ten sessions of the course. The students were grouped into seven groups for the purposes of the Teaching Module. Each group had a Voyage ${ }^{\mathrm{TM}} 200$ calculator that could be used as deemed necessary. The material used for analysis was the recordings (video and audio) of the work of each group in each of the working sessions, in addition to their production on paper and with the calculator.

The analysis of the conceptual and cognitive processes shown by the students was conducted in stages as follows: understanding the problem, construction, analysis, interpretation and generalization of the mathematical model and the retrospective analysis of the solution process. The relationship between the concept of the derivative of a function and the differential equation, general and particular solutions of the ODE that models the problem, its graphical representation and asymptotic behavior were considered for the analysis of the conceptual aspects. A distinction was made between processes, procedures and heuristics within the cognitive processes.

The analysis of data collected showed that both the design of the activity and the environment in which the Teaching Module was developed favored the development of mathematical processes. Thus, the design of the activity permitted the students exhibit mathematical processes such as analysis, representation, resolution, interpretation, generalization or abstraction. The interactions between the students favored the processes of reflection and analysis, as well as the processes of argumentation and the generation of ideas within the resolution of a problem.

It was found that the design of the problem encouraged the students to obtain the expression of an ODE, based on the meaning of the derivative as an element that describes the variation of a function with respect to the independent variable. This also made the students reflect on the mathematical expression obtained and the elements that characterize it, before thinking about the resolution process. The posed hypothetical situation and the activities included as part of the design of the problem permitted the presentation of other mathematical concepts and their use in solving the problem. One can conclude that interactions among students functioned as a control tool for knowledge and for promoting processes such as reflection and argumentation. Finally, the use of technology was key to the development of advanced mathematical thinking processes, for example, the process of generalization, as it allowed students to inquire about more general possible mathematical models, formulate conjectures and verify the assumptions made during the problem solving process. The use of the Voyage ${ }^{\mathrm{TM}} 200$ calculator also facilitated the students to focus on the analysis of the mathematical model from the graphical representation of the solution function of an initial value problem and to establish relationships between different representations of the same mathematical concept. In this way, technology became a natural element in the problem solving process. 
